\title{
Monogamy Equalities for Qubit Entanglement from Lorentz Invariance
}

\author{
Christopher Eltschka \\ Institut für Theoretische Physik, Universität Regensburg, D-93040 Regensburg, Germany \\ Jens Siewert \\ Departamento de Química Física, Universidad del País Vasco-Euskal Herriko Unibertsitatea, 48080 Bilbao, Spain \\ and IKERBASQUE, Basque Foundation for Science, 48011 Bilbao, Spain
}

(Received 29 July 2014; published 7 April 2015)

\begin{abstract}
A striking result from nonrelativistic quantum mechanics is the monogamy of entanglement, which states that a particle can be maximally entangled only with one other party, not with several ones. While there is the exact quantitative relation for three qubits and also several inequalities describing monogamy properties, it is not clear to what extent exact monogamy relations are a general feature of quantum mechanics. We prove that in all many-qubit systems there exist strict monogamy laws for quantum correlations. They come about through the curious relationship between the nonrelativistic quantum mechanics of qubits and Minkowski space. We elucidate the origin of entanglement monogamy from this symmetry perspective and provide recipes to construct new families of such equalities.
\end{abstract}

DOI: 10.1103/PhysRevLett.114.140402

Introduction.-Monogamy of entanglement as a qualitative concept has been discussed for almost two decades $[1,2]$. Conceivably, this intuition can be cast into a mathematical framework of inequalities for certain types of quantum correlations. An influential result is the strong subadditivity of the von Neumann entropy [3], among other important examples for monogamy inequalities [4-9]. Applications of entanglement monogamy pervade many areas of physics, such as quantum information and the foundations of quantum mechanics [10-12], condensedmatter physics [13-15], statistical mechanics [10], and even black-hole physics $[16,17]$.

This has to be contrasted with the possibility of monogamy equalities, i.e., exact relations for different types of correlations in arbitrary pure quantum states. Although several such equalities are known (we mention them explicitly below), the only widely recognized - and at the same time perhaps the most famous-result is the threequbit monogamy relation discovered by Coffman, Kundu, and Wootters (CKW) [18]; cf. Fig. 1.

The existence of monogamy inequalities appears not entirely unexpected, because intuitionally one would associate them with convexity properties of the quantummechanical state space. As opposed to this, rigorous monogamy expressed in terms of equalities for pure states represent a much stronger constraint and hint at a finetuning of the mathematical properties of quantum states. In fact, one might be tempted to use different names for equalities versus inequalities, instead of terming them both "monogamy relations."

In this Letter, we show for qubit systems that such relations are not coincidental, but represent a universal feature of single-copy entanglement that is deeply rooted in
PACS numbers: 03.65.Ud, 03.65.Aa, 03.65.Fd, 03.67.Mn

the algebraic structure of quantum theory. Since exact monogamy relations link those properties to local $\operatorname{SL}(2, \mathbb{C})$ invariants $[19,20]$, they confirm the central importance of these quantities for entanglement theory. Note that in such relations regarding single-copy entanglement, one would not expect entropy-related quantities, such as entanglement of formation encountered in Refs. [3,4,7,8].

The quantum-mechanical state $\rho_{A B C \ldots}$ shared between parties $A, B, C, \ldots$ contains all available information about the correlations between the individual parties, whereas the reduced state, for example, $\rho_{A C(B \cdots)} \equiv \rho_{A C}$, describes the state of the subset $\{A C\}$ of the partners and "forgets" about the information regarding the other parties. For a multipartite system there are many ways to form subsets of parties, and the corresponding reduced states. We may quantify the entanglement contained in each reduced state

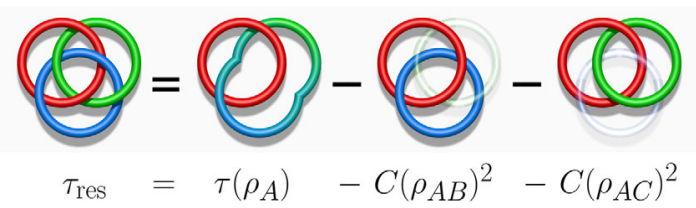

FIG. 1 (color online). Qualitative sketch of the CKW monogamy equality for pure states of three qubits $A, B, C$. The Borromean rings on the left-hand side adequately illustrate the tripartite entanglement quantified by $\tau_{\text {res. }}$ The first term on the right-hand side stands for the linear entropy $\tau\left(\rho_{A}\right) \equiv \tau\left(\rho_{A(B C)}\right)$ of qubit $A$ that measures the entanglement between $A$ and the composite system $\{B C\}$. The remaining two terms represent the amount of bipartite entanglement of $A$ with $B$ or $C$ quantified by the concurrences $C\left(\rho_{A B}\right)^{2}$ and $C\left(\rho_{A C}\right)^{2}$, respectively, thereby "forgetting" (tracing out) the third party. While $\tau_{\text {res }}$ is a global property of the state, the quantities on the right-hand side refer to different reduced states. 
by an appropriate entanglement measure. A monogamy relation is nothing but a mathematical constraint for the entanglement quantifiers of different reduced states of a composite quantum system (cf. Fig. 2).

Bloch representation and Lorentz invariance.-Usually, nonrelativistic quantum mechanics is constructed starting from pure states in Hilbert space, which later are generalized to mixed states representing ensembles of pure states. In our work, we emphasize the opposite point of view, that is, that states are positive Hermitian operators (or "density matrices") and pure states are special states characterized by additional conditions. It is those conditions that imply peculiar properties, such as strict monogamy of entanglement. In order to see this, it is essential to choose a representation for the density matrices that adequately encodes the relevant state properties, the Bloch representation [21-24].

Consider the state $\rho$ of a single qubit, which can be written as [21-24]

$$
\rho=\frac{1}{2}\left(r_{0} \mathbb{1}_{2}+r_{1} \sigma_{1}+r_{2} \sigma_{2}+r_{3} \sigma_{3}\right),
$$

where $\sigma_{j}$ are the Pauli matrices, $\mathbb{1} \equiv \sigma_{0}$ is the identity operator for qubits, and $r_{j}$ are real numbers. Usually, $\rho$ is normalized so that $r_{0}=1$. An important quantity is the determinant [22],

$$
4 \operatorname{det} \rho=2 \operatorname{tr} \rho \sigma_{2} \rho^{T} \sigma_{2}=r_{0}^{2}-r_{1}^{2}-r_{2}^{2}-r_{3}^{2} \equiv r_{\mu} r^{\mu},
$$

because it does not change under determinant-one operations $F \in \operatorname{SL}(2, \mathbb{C}): \rho \rightarrow F \rho F^{\dagger}$. We have introduced the Einstein summation convention $r_{\mu} r^{\mu} \equiv \sum_{\mu, \nu} \eta^{\mu \nu} r_{\mu} r_{\nu}$ and the Minkowski metric $\eta^{\mu \nu}=\operatorname{diag}(1,-1,-1,-1)$. Note that this Minkowski metric acts on Bloch space, not on physical spacetime. Also, the terms "spacelike" and "timelike" used below refer to the Lorentz group structure found in Bloch space, not to physical space and time. As is known from Lie theory, local SL invariance translates into Lorentz invariance in the Bloch coefficients $r_{\mu}$. This representation can be generalized to any number $N$ of qubits

$$
\rho=\frac{1}{2^{N}} \sum_{j_{1}, \ldots, j_{N}} r_{j_{1} j_{2} \cdots j_{N}} \sigma_{j_{1}} \otimes \cdots \otimes \sigma_{j_{N}}
$$

as well as

$$
\operatorname{tr} R \equiv \operatorname{tr} \rho \sigma_{2}^{\otimes N} \rho^{T} \sigma_{2}^{\otimes N}=\frac{1}{2^{N}} r_{\mu_{1} \cdots \mu_{N}} r^{\mu_{1} \cdots \mu_{N}}
$$

(using the transposed density matrix $\rho^{T}$ ). This expression is invariant under local determinant-one operations implying Lorentz invariance independently on each qubit index [22]. It is also non-negative since $R$ can be rearranged under the trace as a positive operator [25] $\operatorname{tr} R=\operatorname{tr} \sqrt{\rho} \sigma_{2}^{\otimes N} \rho^{T} \sigma_{2}^{\otimes N} \sqrt{\rho}$. The aforementioned relations (2) and (4) are valid for arbitrary states, but we may ask whether for pure states more elaborate predictions are possible.

Origin of exact monogamy.-The space of all states $\rho$ is a convex set, with the pure states $\pi_{\psi}$ as extreme points. They can be characterized as projectors

$$
\pi_{\psi}=\pi_{\psi}^{2} .
$$

Here, $\psi$ refers to the usual bra-ket notation, that is, $\pi_{\psi}=|\psi\rangle\langle\psi|$. Inserting Eq. (3) into Eq. (5) yields an operator identity that has to be satisfied termwise. The most prominent among these conditions is the normalization of $\pi_{\psi}^{2}$,

$$
\operatorname{tr} \pi_{\psi}^{2}=1=\frac{1}{2^{N}} \sum r_{\mu_{1} \cdots \mu_{N}}^{2} .
$$

Surprisingly, this is enough to explain the origin of monogamy equalities. To this end, we write the Minkowskian and Euclidean sums (4) and (6) explicitly, separating timelike and spacelike indices (we show an example for two qubits),

$$
\begin{aligned}
2^{2} \operatorname{tr} R_{\psi} & =r_{00}^{2}-\sum_{j=1}^{3}\left(r_{0 j}^{2}+r_{j 0}^{2}\right)+\sum_{j, k=1}^{3} r_{j k}^{2} \\
& \equiv S_{0}-S_{1}+S_{2}, \\
2^{2} \operatorname{tr} \pi_{\psi}^{2} & =S_{0}+S_{1}+S_{2} .
\end{aligned}
$$

The symbol $S_{k}$ denotes the sum of all terms $r_{\ldots}^{2}$. with $k$ spacelike indices. Each quantity $S_{k}$ is invariant under local unitaries. The coefficients $r_{\text {... with a timelike index } 0 \text { at }}$ position $q$ are components of a reduced state $\operatorname{tr}_{q} \pi_{\psi}$. Finally, for pure states we have $\operatorname{tr} R_{\psi}=|H(\psi)|^{2}$ with the wellknown polynomial invariant $[19,26,27] H(\psi)$, i.e., a quantity that characterizes and quantifies global entanglement in the pure state $\pi_{\psi}$.

It is evident from Eqs. (8) and (9) that the term $S_{2}$ with only spacelike indices can be eliminated, so that we are left with an equation that contains only quantities that either characterize global entanglement in the state or describe elements of reduced states. This is the general monogamy principle that obviously works for any number of qubits, since the corresponding relations for purity and $|H(\psi)|^{2}$ are always independent. The question is whether and how the terms of the reduced states can be related to entanglement measures in a simple manner.

Degree-2 monogamy relations. - In the following, we show how specific monogamy relations can be deduced from this principle. By either subtracting (even $N$ ) or adding (odd $N$ ) the equations for $\operatorname{tr} R$ and $\operatorname{tr} \rho^{2}$, the term with only spacelike indices is eliminated and we obtain an equality for all $N$-qubit mixed states, 


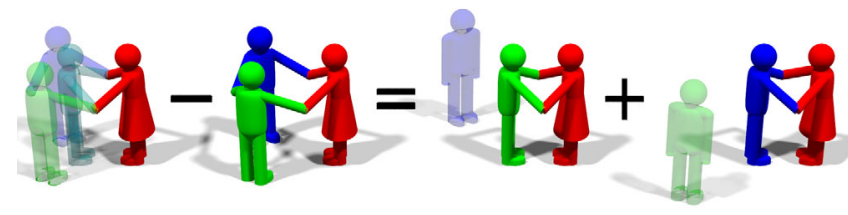

FIG. 2 (color online). Why is the CKW equality in Fig. 1 termed a "monogamy relation"? The linear entropy $\tau\left(\rho_{A}\right)$ can be viewed as the total amount of Alice's social connectivity (first picture on the left), while the residual tangle $\tau_{\text {res }}$ represents connectivity in a group that Alice, Bob, and Charlie share symmetrically (second picture on the left). On the other hand, the concurrences $C\left(\rho_{A B}\right)^{2}, C\left(\rho_{A C}\right)^{2}$ characterize Alice's personal relation with Bob or Charlie, respectively. As the equality shows, if Alice's total connectivity and the group connectivity are fixed, so is the sum of her personal relations. That is, Alice may share relations with Bob and Charlie, but she cannot dedicate maximal attention to both Bob and Charlie at the same time.

$$
(-1)^{N} \operatorname{tr} R=\operatorname{tr} \rho^{2}-\frac{1}{2^{N-1}} \sum_{k=a_{N}}^{\lfloor N / 2\rfloor} S_{2 k-a_{N}},
$$

where $\lfloor N / 2\rfloor$ denotes the largest integer not exceeding $N / 2$ and $a_{N}=\frac{1}{2}\left[1+(-1)^{N}\right]$. The sums $S_{k}$ can be expressed in terms of the purities $\operatorname{tr} \rho_{\{j\}}^{2}$ of the reduced density matrices $\rho_{\{j\}}$ with a set of $j$ qubits traced out. The purities are related to the linear entropy $\tau(\rho)=2\left[(\operatorname{tr} \rho)^{2}-\operatorname{tr} \rho^{2}\right]$, so that

$$
(-1)^{N} \operatorname{tr} R=-1+\operatorname{tr} \rho^{2}+\frac{1}{2} \sum_{j=1}^{N-1}(-1)^{j+1} \tau_{(j)} .
$$

Here, $\tau_{(j)} \equiv \sum_{\{j\}} \tau\left(\operatorname{tr}_{\{j\}} \rho\right)$ is the sum of all linear entropies that are obtained by tracing out $j$ qubits from the state $\rho$. For pure states $\psi$ the linear entropy equals the squared concurrence (for each bipartition $A \mid B$ )

$$
\tau\left(\operatorname{tr}_{B} \pi_{\psi}\right)=\tau\left(\operatorname{tr}_{A} \pi_{\psi}\right)=C_{A \mid B}^{2}(\psi),
$$

and we can straightforwardly derive an exact monogamy relation for all integers $N \geqq 2$,

$$
\begin{aligned}
2|H(\psi)|^{2}= & \sum_{\{1\}} C_{\{1\} \mid\{N-1\}}(\psi)^{2}-\sum_{\{2\}} C_{\{2\} \mid\{N-2\}}(\psi)^{2} \\
& +\sum_{\{3\}} C_{\{3\} \mid\{N-3\}}(\psi)^{2}-+\cdots \\
& +(-1)^{N} \sum_{\{N-1\}} C_{\{N-1\} \mid\{1\}}(\psi)^{2}
\end{aligned}
$$

where $C_{\{j\} \mid\{N-j\}} \equiv \sqrt{\tau\left(\operatorname{tr}_{\{j\}} \rho\right)}$ and $\sum_{\{j\}}$ is taken over all combinations $\{j\}$ of $j$ indices. Note that the right-hand side of Eq. (13) is a combination of entanglement monotones on the respective bipartitions. For $N=4$, this relation was found in Ref. [28].
For odd qubit number, $\operatorname{tr} R_{\psi}=|H(\psi)|^{2} \equiv 0$, which leads to an identity because

$$
\operatorname{tr}_{B}\left(\operatorname{tr}_{A} \pi_{\psi}\right)^{2}=\operatorname{tr}_{A}\left(\operatorname{tr}_{B} \pi_{\psi}\right)^{2}
$$

[and, hence, $C_{A \mid B}(\psi)=C_{B \mid A}(\psi)$ ] for any bipartition $A \mid B$ in a pure state.

Thus, we have found the simplest monogamy relations which contain, in a sense, averaged quantities (cf. Fig. 3). For example, for two qubits $A$ and $B$ the invariant polynomial equals Wootters' concurrence [25] $|H(\psi)|=C(\psi), \quad$ so $\quad$ that $\quad 2 C(\psi)^{2}=\sum_{\{1\}} C_{\{1\} \mid\{1\}}^{2}=$ $C_{A \mid B}^{2}+C_{B \mid A}^{2}$, while obviously $C(\psi)=C_{A \mid B}(\psi)=C_{B \mid A}(\psi)$. In order to obtain these sharper relations, one can use Eq. (5). Nonetheless, Eq. (13) is remarkable: On the righthand side, all terms are invariant under local unitaries while the left-hand side is $\operatorname{SL}(2, \mathbb{C})$ invariant on all parties. This is one of the hallmarks of equalities for entanglement monogamy. Notably, the relation is homogeneous in the state (more precisely, of degree 2 in the density matrix).

Degree-4 monogamy relations.-Our strategy for obtaining monogamy relations so far was as follows: consider a local SL invariant expression for the state, reveal its Lorentz-invariant structure, and rewrite it in terms of the reduced states, thereby eliminating the exclusively spacelike parts. Until now we have considered only the invariant $H(\psi)$, which is of homogeneous degree 2 in the coefficients of the Hilbert vector $\psi$. In the next step, we investigate degree-4 invariants, which will lead us also to the CKW monogamy relation.

A local SL invariant of degree 4 deriving from the $\mathcal{B}^{(N)}$ invariants for odd $N$ qubits in Ref. [27] is

$$
\left(\operatorname{tr} \rho \Sigma_{\gamma_{1}} \rho^{T} \Sigma_{\gamma_{2}}\right)\left(\operatorname{tr} \rho \Sigma^{\gamma_{1}} \rho^{T} \Sigma^{\gamma_{2}}\right) \equiv B_{C}^{(3)}(\rho),
$$

with $\Sigma_{\gamma} \equiv \sigma_{2} \otimes \sigma_{2} \otimes \sigma_{\gamma}$. Pure states satisfy $B_{C}^{(3)}(\psi)=$ $\left|\mathcal{B}_{C}^{(3)}(\psi)\right|^{2}$. Here, we also define the $\mathcal{B}^{(N)}$ invariants with the full Minkowski metric (as opposed to Ref. [27]). We obtain

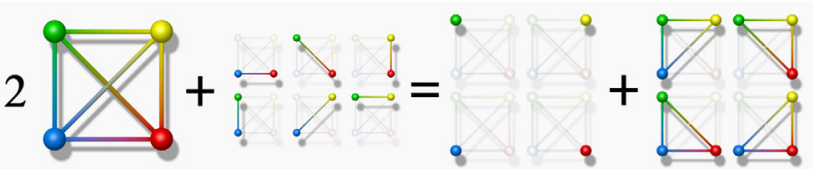

FIG. 3 (color online). Illustration of the degree-2 monogamy relation for pure four-qubit states. The big square on the left represents $|H(\psi)|^{2}$ while the other items stand for the six possibilities of two-qubit concurrences. Correspondingly, on the right-hand side, the sums of all concurrences related to single-qubit and three-qubit bipartitions are shown. Note that the terms on the right are pairwise identical according to Eq. (14). For example, the single-qubit concurrence of the "green qubit" equals that of the other three qubits (lower right entry of the three-qubit contributions). 


$$
B_{C}^{(3)}(\psi)=\frac{1}{2^{4}} r_{\alpha \beta \gamma} r^{\alpha \beta \nu} r_{\lambda \mu \nu} r^{\lambda \mu \gamma}=\tau_{\text {res }}(\psi)^{2} .
$$

In order to eliminate the spacelike indices on qubit $C$, we use the following pure-state identities, which can be verified using the Schmidt decomposition. Abbreviating $\tilde{\pi}_{\psi} \equiv \Sigma_{2} \pi_{\psi}^{T} \Sigma_{2}$, we have

$$
\begin{aligned}
\left(\operatorname{tr} R_{A B}\right)^{2} & =\operatorname{tr}\left[\pi_{\psi}\left(\operatorname{tr}_{C} \tilde{\pi}_{\psi} \operatorname{tr}_{A B} \tilde{\pi}_{\psi}\right) \pi_{\psi}\right], \\
\operatorname{tr} R_{A B}^{2} & =\operatorname{tr}\left[\left(\operatorname{tr}_{C} \pi_{\psi}\right) \tilde{\pi}_{\psi} \operatorname{tr}_{A B}\left(\operatorname{tr}_{C} \pi_{\psi}\right) \tilde{\pi}_{\psi}\right],
\end{aligned}
$$

where the rightmost trace is taken first and traces have to be read such that their argument extends all the way to the right, except for the $C$ traces, which remain within their parentheses. Thus,

$$
\begin{aligned}
\left|\mathcal{B}_{C}^{(3)}(\psi)\right|^{2} & =8\left[\left(\operatorname{tr} R_{A B}\right)^{2}-\operatorname{tr} R_{A B}^{2}\right] \\
& =4\left[\operatorname{tr} R_{A B}-C\left(\rho_{A B}\right)^{2}\right]^{2},
\end{aligned}
$$

where $C\left(\rho_{A B}\right)$ is the concurrence of the rank-2 state $\rho_{A B}$ and the calculation follows the spirit of Ref. [18]. For $N>3$, all derivations are completely analogous and we obtain

$$
\left|\mathcal{B}_{j}^{(N)}(\psi)\right|=2\left[\operatorname{tr} R_{[j]}-\left|H\left(\operatorname{tr}_{j} \pi_{\psi}\right)\right|^{2}\right],
$$

where $\operatorname{tr} R_{[j]} \equiv \operatorname{tr} R\left(\operatorname{tr}_{j} \pi_{\psi}\right)$ and $\left|H\left(\operatorname{tr}_{j} \pi_{\psi}\right)\right|$ is the convex roof of $|H|$ for the rank-2 state $\operatorname{tr}_{j} \pi_{\psi}$ according to the Wootters-Uhlmann method [25,29]. Further, $\operatorname{tr} R_{[j]}$ may be replaced using Eq. (11). The simplest way to get a quartic monogamy equality like Eq. (13) is to add the relations (17) for the $\left|\mathcal{B}_{j}^{(N)}\right|$ of all qubits,

$$
\begin{aligned}
& \sum_{j=1}^{N}\left[\left|\mathcal{B}_{j}^{(N)}(\psi)\right|+2\left|H\left(\operatorname{tr}_{j} \pi_{\psi}\right)\right|^{2}\right] \\
& \quad=(-1)^{N} \sum_{j=1}^{N-1}(-1)^{j+1} j \sum_{\{j\}} C_{\{j\} \mid\{N-j\}}(\psi)^{2} .
\end{aligned}
$$

We mention that CKW equality is obtained by adding the relations for $\mathcal{B}_{C}^{(3)}$ and $\mathcal{B}_{B}^{(3)}$ only.

Curiously, for even $N$ the quartic monogamy equality is not strictly of degree 4 because $\left|\mathcal{B}_{j}^{(2 m)}(\psi)\right|=|H(\psi)|^{2}$, and by using Eq. (14) we get back to Eq. (13), since all terms $\left|H\left(\operatorname{tr}_{j} \pi_{\psi}\right)\right|$ vanish. Yet, there do exist degree-4 monogamy equalities also for $N=4$ (cf. Ref. [27]); e.g.,

$$
\left|\mathcal{B}_{12}^{(4)}(\psi)-\mathcal{B}_{13}^{(4)}(\psi)\right|^{2}=48^{2} \operatorname{det}\left(\operatorname{tr}_{14} \pi_{\psi}\right) .
$$

Discussion.-We have derived degree-2 and degree-4 monogamy relations of pure-state qubit entanglement, the central results being Eqs. (13) and (18). We emphasize that on both sides of these equalities, there appear only polynomial functions of the Bloch coefficients. Consequently, these relations can contain only entanglement measures based on polynomial functions, and therefore describe single-copy entanglement properties. They can be interpreted as follows. Tracing out $B$ in a bipartition $A \mid B$ removes the coherences between $A$ and $B$, thus converting their mutual quantum correlations into classical correlations of $A$ alone. The global accounting of all these correlations, which are quantified by the concurrences, is given by the $\operatorname{SL}(2, \mathbb{C})^{\otimes N}$ invariants on the left-hand side of Eqs. (13) and (18). The right-hand sides can be viewed as decompositions of the global into bipartite correlations. We mention that for qubit systems, there are algebraically independent local SL invariants also of degree 6 (for $N \geqq 4$ ) as well as of degree 8,10 , etc. $(N>4)$. We expect that it is possible to find corresponding monogamy equalities also for those invariants by continuing the hierarchy described in this Letter.

This work was funded by the German Research Foundation within SPP 1386 (C. E.), by Basque Government Grant No. IT-472-10, MINECO Grant No. FIS2012-36673-C03-01, and Universidad del Pais Vasco/Euskal Herriko Unibertsitatea (UPV/EHU) Program No. UFI 11/55 (J. S.). The authors thank A. Uhlmann for helpful remarks and J. Fabian, J. G. Muga, and K. Richter for their support.

[1] C. H. Bennett, D. P. DiVincenzo, J. A. Smolin, and W. K. Wootters, Phys. Rev. A 54, 3824 (1996).

[2] B. Terhal, IBM J. Res. Dev. 48, 71 (2004).

[3] E. H. Lieb and M. B. Ruskai, J. Math. Phys. (N.Y.) 14, 1938 (1973).

[4] M. Koashi and A. Winter, Phys. Rev. A 69, 022309 (2004).

[5] T. J. Osborne and F. Verstraete, Phys. Rev. Lett. 96, 220503 (2006).

[6] Y.-C. Ou, H. Fan, and S.-M. Fei, Phys. Rev. A 78, 012311 (2008).

[7] T. R. de Oliveira, M. F. Cornelio, and F. F. Fanchini, Phys. Rev. A 89, 034303 (2014).

[8] Y.-K. Bai, Y.-F. Xu, and Z. D. Wang, Phys. Rev. Lett. 113, 100503 (2014).

[9] B. Regula, S. Di Martino, S.-J. Lee, and G. Adesso, Phys. Rev. Lett. 113, 110501 (2014).

[10] C. H. Bennett, in Proceedings of the FQXi 4th International Conference, Vieques Island, Puerto Rico, 2014, http://fqxi .org/conference/talks/2014.

[11] B. Toner, Proc. R. Soc. A 465, 59 (2009).

[12] M. P. Seevinck, Quantum Inf. Process. 9, 273 (2010).

[13] X.-s. Ma, B. Dakic, W. Naylor, A. Zeilinger, and P. Walther, Nat. Phys. 7, 399 (2011).

[14] F. G. S. L. Brandao and A. W. Harrow, in Proceedings of the 45th Annual ACM Symposium on Theory of Computing, 2013, http://dl.acm.org/citation.cfm?doid=2488608 .2488718 .

[15] A. García-Sáez and J. I. Latorre, Phys. Rev. B 87, 085130 (2013). 
[16] L. Susskind, arXiv:1301.4505.

[17] S. Lloyd and J. Preskill, J. High Energy Phys. 08 (2014) 126.

[18] V. Coffman, J. Kundu, and W. K. Wootters, Phys. Rev. A 61, 052306 (2000).

[19] J.-L. Brylinski and R. Brylinski, in Mathematics of Quantum Computation, edited by G. Chen and R.-K. Brylinski (Chapman and Hall, London, 2002), Chap. 11.

[20] F. Verstraete, J. Dehaene, and B. De Moor, Phys. Rev. A 68, 012103 (2003).

[21] F. T. Hioe and J. H. Eberly, Phys. Rev. Lett. 47, 838 (1981).

[22] M. Teodorescu-Frumosu and G. Jaeger, Phys. Rev. A 67, 052305 (2003).
[23] G. Mahler and V. A. Weberruß, Quantum Networks, 2nd ed. (Springer, Berlin, 2004).

[24] I. Bengtsson and K. Zyczkowski, Geometry of Quantum States (Cambridge University Press, Cambridge, England, 2006).

[25] W. K. Wootters, Phys. Rev. Lett. 80, 2245 (1998).

[26] A. Wong and N. Christensen, Phys. Rev. A 63, 044301 (2001).

[27] C. Eltschka, T. Bastin, A. Osterloh, and J. Siewert, Phys. Rev. A 85, 022301 (2012); 85, 059903(E) (2012).

[28] G. Gour and N. R. Wallach, J. Math. Phys. (N.Y.) 51, 112201 (2010).

[29] A. Uhlmann, Phys. Rev. A 62, 032307 (2000). 